International Journal of Current Advanced Research

ISSN: O: 2319-6475, ISSN: P: 2319 - 6505, Impact Factor: SJIF: 5.995

Available Online at www.journalijcar.org

Volume 6; Issue 3; March 2017; Page No. 2389-2393

DOI: http://dx.doi.org/10.24327/ijcar.2017.2393.0015

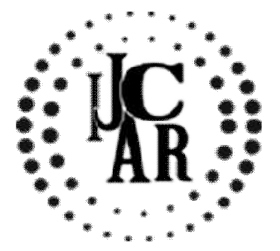

Research Article

\title{
EVALUATION OF POSITIONAL ACCURACY OF EPID USING IMRT GRATICULE PHANTOM IN EXTENDED SOURCE TO IMAGER DISTANCE SETUPS: FORMALISM OF QA
}

\author{
Ranjit Singh ${ }^{1,3}{ }^{*}$, Teerthraj Verma ${ }^{2}$., Harpreet Singh Kainth ${ }^{3}$, Chappe Ram ${ }^{4}$, Devinder Mehta ${ }^{3}$, \\ Bhupinder S. Rana ${ }^{5}$, Jangvir Singh Shahi ${ }^{3}$ and Baljinder Singh ${ }^{6}$
}

${ }^{1}$ Department of Radiotherapy, Post Graduate Institute of Medical Education \& Research, Chandigarh, India

${ }^{2}$ Department of Radiological Physics, King George Medical University, Lucknow, U.P., India

${ }^{3}$ Physics Department, Panjab University, Chandigarh, India

${ }^{4}$ Department of Radiotherapy \& Radiation Medicine, Banaras Hindu University, Varanasi, India

${ }^{5}$ Department of Diagnosis and Imaging, Post Graduate Institute of Medical Education \& Research, Chandigarh, India

${ }^{6}$ Department Nuclear Medicine, Post Graduate Institute of Medical Education \& Research, Chandigarh, India

\section{A R T I C L E I N F O \\ Article History: \\ Received $11^{\text {th }}$ December, 2016 \\ Received in revised form $24^{\text {th }}$ January, 2017 \\ Accepted $10^{\text {th }}$ February, 2017 \\ Published online $28^{\text {th }}$ March, 2017}

\section{Key words:}

Graticule Phantom, IMRT QA, EPID, extended SID.

\begin{abstract}
A B S T R A C T
Purpose: The aim of the present study was to evaluate the utility of IMRT graticule phantom to check the positional accuracy of EPID (amorphous silicon flat panel detector, retractable arm) and to develop a quality assurance program for geometrical verification. Method: The radiographic images of graticule phantom were acquired using computed tomography (CT) and beam shapes for desired dose distribution was generated using computer based treatment planning system ( Ecllipse, version 8.6, Varian, Palo, alto, CA). A known shift of $0.5 \mathrm{~cm}, 1.0 \mathrm{~cm}$ and $1.5 \mathrm{~cm}$ were introduced in longitudinal, lateral and vertical directions, respectively w.r.t. treatment couch of medical linear accelerator. The EPID images were taken for each shift at different source to imager distance (SID) and beam orientations.

Results: The maximum and minimum shift between the expected and observed value in all the direction were found to be $3 \mathrm{~mm}$ and zero respectively. In longitudinal and vertical directions, maximum error of $2 \mathrm{~mm}$ was obtained for SID $179.9 \mathrm{~cm}$ and $149.9 \mathrm{~cm}$, respectively, while in lateral direction the $2 \mathrm{~mm}$ maximum error was obtained for imager distance $149.9 \mathrm{~cm}$ and $179.9 \mathrm{~cm}$. However, the maximum error of $3 \mathrm{~mm}$ was found to be most frequent in the longitudinal and vertical directions for SID $149.9 \mathrm{~cm}$ and $179.9 \mathrm{~cm}$, respectively.

Conclusion: The methodology used in the present study is very effective to check the mechanical characteristic and consistency of the retractable arm EPID and can be used routinely in radiotherapy units. The effect of EPID sag due to gravity was not significant for detection of shift in patient's position.
\end{abstract}

Copyright $₫ 2017$ Ranjit Singh et al. This is an open access article distributed under the Creative Commons Attribution License, which permits unrestricted use, distribution, and reproduction in any medium, provided the original work is properly cited.

\section{INTRODUCTION}

The recent advancement in radiotherapy techniques requires accurate dose delivery and reproducible patient's position setup during treatment execution. The benefits of advanced treatment modalities could only be derived if the planned and delivered dose distribution matches exactly with each other. A small mismatch between these two may results in increase normal tissue complications and decrease in the tumor control probability $^{1-4}$. Earlier X-ray port films were used in radiotherapy treatment to verify day to day patient's setup

*Corresponding author: Ranjit Singh

Department of Radiotherapy, Post Graduate Institute of Medical Education \& Research, Chandigarh, India which was time consuming and labor intensive and hence reduces patient throughput in the busy radiotherapy centre. ${ }^{5}$ The introduction of electronic portal imaging device (EPID) in radiotherapy overcomes these problems. These are used for verification of patient position as well as dosimetric verification in complex radiotherapy modalities, e.g., intensity modulated radiotherapy (IMRT), image guided radiotherapy (IGRT) etc. ${ }^{6-7}$ and quality assurance (QA) of medical linear accelerator. These treatment modalities (IMRT, IGRT) delivers non-uniform dose fluence with rapid dose fall off at the edge of the tumor volume. EPID is very effective for invivo dose measurements for such type of treatment ${ }^{8-9}$ and the data analysis is rapid because of faster image acquisition and greater spatial resolution. ${ }^{10}$ The task of multiple images, intra and inter-fractional assessment, or quantitative analysis of 3D treatment setup parameters were easily performed using 


\section{Evaluation of positional accuracy of epid using imrt graticule phantom in extended source to imager distance setups: formalism of qa}

EPID. ${ }^{11-12}$ The increase in frequency of imaging reduces the day to day patient error and hence improves the treatment precision. ${ }^{12}$ The successful implementation of EPID in clinical oncology requires careful and regular QA and maintenance of EPID devices. ${ }^{13}$ The QA of linear accelerator, e.g., performance, positional accuracy, inter and intra leaf radiation leakage of multi leaf collimators (MLC), field size verification, jaws position and orthogonality check etc. were also performed using EPID. The exclusive use of EPID based QA tools, including a QA phantom and simultaneous analysis software tools has been demonstrated as a viable, efficient, and comprehensive process for daily evaluation of LINAC performance. ${ }^{14}$ The commercially available EPID technology system consist of either matrix ion chamber or camera-based EPIDs or latest introduced active matrix flat panel imaging (AMFPI) and their properties are extensively studied by different authors. ${ }^{15-18}$ The aim of the present study is to evaluate the utility of IMRT graticule phantom to check the positional accuracy of EPID (amorphous silicon flat panel detector, retractable arm) and develop a QA program for geometrical verification.

\section{MATERIAL AND METHOD}

In the present work, a Medical Linear Accelerator (Linac), Clinac DBx -1160 (Varian, Palo, alto, CA) from Varian medical system (Fig.1) having photon energy of $6 \mathrm{MeV}$ was used. The Linear Accelerator ${ }^{19-20}$ is equipped with a-Si 500 portal vision image detectors attached to the machine by retractable supporting arm (R-arm) for portal imaging.

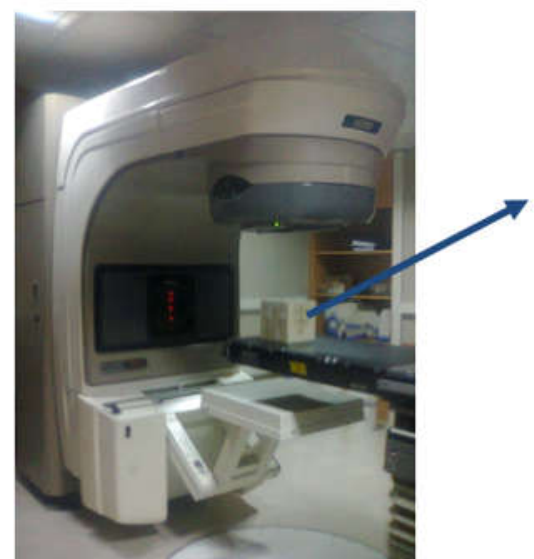

\section{Description of $Q A$ Test}

The IMRT graticule phantom was placed on the couch of Computed Tomography (CT) simulator and three lead ball markers were placed on the respected faces at the point of intersection of all the three lasers (longitudinal, vertical and horizontal). The radiographic images of the phantom were acquired on the CT simulator with slice thickness of $2.5 \mathrm{~mm}$. These CT images were sent to the planning system through digital communication (DICOM) and treatment plan was made using PBC algorithm.

The setup fields for anterior, left lateral and right lateral portal images were added to the existing plan. Digitally reconstructed radiographs (DRR) of the phantom were generated for each setup field on the TPS system (eclipse) and were considered as reference images. IMRT phantom was placed on the couch of the machine in a position reproducible as during the time of $\mathrm{CT}$ image acquisition. The appropriate shift obtained from the TPS plan was applied in longitudinal, lateral and vertical directions, so that the centre of irradiated volume coincides with machine isocentre. This position was considered as the reference position of the given IMRT phantom. The EPID images were taken for anterior, left lateral and right lateral setup of the same phantom. For a patient laid down in supine or prone position, the anterior portal image will give variation in the lateral ( $\mathrm{x}$-axis) and longitudinal (y-Axis) direction while the left lateral and right lateral portals give changes in the longitudinal and vertical

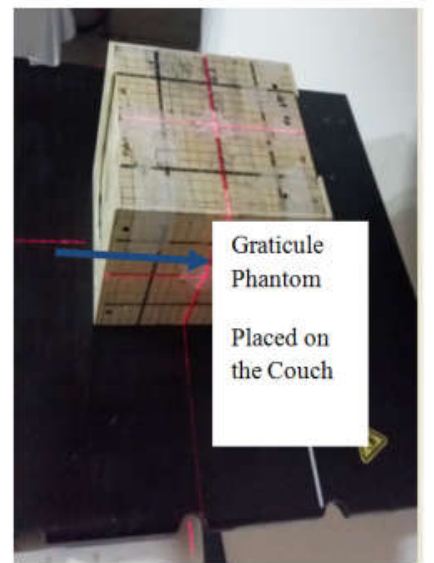

Fig. 1 Experimental setup (I) showing graticule phantom (II) placed over treatment couch of Clinax DBX-1160 along with inbuilt portal vision A-Si 500 (supported with retractable arm) for acquiring radiographic images.

The computer based treatment planning system, Ecllipse (version 8.6) uses pencil beam convolution (PBC) ${ }^{21-22}$ and analytical anisotropic algorithms ${ }^{23-24}$ with selective calculation grid size. The beam shapes and desired dose distributions are generate with the aim to maximize tumor control and minimize normal tissue complications. IMRT graticule phantom used in the present study was made up of tissue equivalent material and has a dimension of $18 \mathrm{~cm} \times 18$ $\mathrm{cm} \times 18 \mathrm{~cm}$. This phantom is quite useful to carry out X-ray port film dosimetry for various treatment modalities where the dose distribution is very complex. This phantom is provided with inbuilt graticule which is made up of radio-opaque markers and clearly visible in radiographic images. direction ( $\mathrm{z}$ axis), respectively. Therefore, combination of anterior portal and any one of lateral portal is sufficient to provide variation in all three directions w. r. t. original planned patient's position. A known shift of $0.5 \mathrm{~cm}, 1.0 \mathrm{~cm}$ and $1.5 \mathrm{~cm}$ were introduced in longitudinal (in and out), lateral (right and left) and vertical (up and down) directions, respectively, and EPID images were acquired for each shift at a particular SID. The measurements were repeated for same shift at varying SID at $140.0 \mathrm{~cm}, 149.9 \mathrm{~cm}, 159.9 \mathrm{~cm}$ and $179.9 \mathrm{~cm}$. These portal images were matched manually on the console monitor as well as offline review on TPS [Fig. 2] and measured shift were recorded for each portal image. The expected shifts and observed shifts are given in Tables 1-3. 


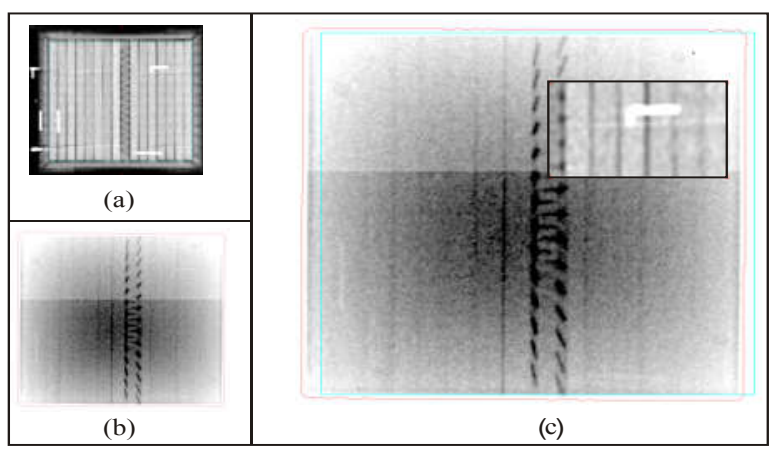

Fig. 2 (a) The anterior DRR image (b) The image acquired from EPID and (c) The blended image obtained during manual match of acquired image (shown in background) and DRR image(shown in inset)

\section{RESULTS}

Table 1 illustrates the expected and observed shifts obtained for different beam angles (gantry angles $0^{\circ}, 90^{\circ}$ and $270^{\circ}$ for anterior, left lateral and right lateral portal images, respectively) in the longitudinal direction at varying SID. The maximum and minimum difference among all shifts in the inward and outward direction was found to be $3 \mathrm{~mm}$ and zero, respectively [Fig. 3(i)].
The maximum error obtained for $179.9 \mathrm{~cm}$ SID was $2 \mathrm{~mm}$. There was no difference between the expected and observed values at imager distance $179.9 \mathrm{~cm}$ for inward shifts obtained in left lateral portal images and outward shifts obtained for right lateral portal images. The frequency of occurrence of maximum error of $3 \mathrm{~mm}$ was highest obtained for imager distance $149.9 \mathrm{~cm}$. Table 2 provides the information of lateral shifts obtained from portal image at $0^{\circ}$ gantry angle (anterior portal image) and the difference between the expected and observed shift in the lateral directions (left and right lateral). The maximum difference between the expected and observed shift was found to be $3 \mathrm{~mm}$ and was obtained both for imager distance 140.0 and $159.9 \mathrm{~cm}$ [Fig. 3 (ii)]. The maximum error obtained were $2 \mathrm{~mm}$ both for imager distances 149.9 and $179.9 \mathrm{~cm}$. Table 3 shows the error obtained in vertical direction for manually introduced shift. The maximum shifts between expected and observed values were found to be 3 $\mathrm{mm}$ and the frequency of occurrence is maximum for imager distance $179.9 \mathrm{~cm}$ both for lateral portal images. There is no difference between observed and expected values of shifts for images acquired from left lateral portals at $149.9 \mathrm{~cm}$ while maximum difference obtained from online correction were 2 $\mathrm{mm}$ for the right lateral portal images [Fig. 3(iii)]. The maximum difference obtained for imager distance $159.9 \mathrm{~cm}$ was $2 \mathrm{~mm}$.

Table 1 The expected and observed (obs.) shifts in the longitudinal direction obtained from different portal image at different SID.

\begin{tabular}{|c|c|c|c|c|c|c|c|c|c|c|c|c|}
\hline \multirow{3}{*}{ SID (cm) } & \multicolumn{4}{|c|}{ Anterior portal image } & \multicolumn{4}{|c|}{ Left lateral portal image } & \multicolumn{4}{|c|}{$\begin{array}{l}\text { Right lateral Portal image } \\
\end{array}$} \\
\hline & \multicolumn{2}{|c|}{ inward shift (cm) } & \multicolumn{2}{|c|}{ outward shift (cm) } & \multicolumn{2}{|c|}{ inward shift (cm) } & \multicolumn{2}{|c|}{ outward shift (cm) } & \multicolumn{2}{|c|}{ inward shift (cm) } & \multicolumn{2}{|c|}{ outward shift (cm) } \\
\hline & Expected & Obs. & Expected & Obs. & Expected & Obs. & Expected & Obs. & Expected & Obs. & Expected & Obs. \\
\hline \multirow{4}{*}{140.0} & -0.5 & -0.7 & 0.5 & 0.4 & -0.5 & -0.7 & 0.5 & 0.5 & -0.5 & -0.3 & 0.5 & 0.6 \\
\hline & -1.0 & -1.1 & 1.0 & 0.9 & -1.0 & -1.1 & 1.0 & 0.9 & -1.0 & -1.1 & 1.0 & 1.0 \\
\hline & -1.5 & -1.8 & 1.5 & 1.4 & -1.5 & -1.3 & 1.5 & 1.4 & -1.5 & -1.8 & 1.5 & 1.5 \\
\hline & -0.5 & -0.5 & 0.5 & 0.4 & -0.5 & -0.5 & 0.5 & 0.4 & -0.5 & -0.3 & 0.5 & 0.8 \\
\hline \multirow[t]{2}{*}{149.9} & -1.0 & -1.3 & 1.0 & 0.8 & -1.0 & -0.9 & 1.0 & 0.7 & -1.0 & -1.0 & 1.0 & 1.1 \\
\hline & -1.5 & -1.4 & 1.5 & 1.3 & -1.5 & -1.8 & 1.5 & 1.3 & -1.5 & -1.5 & 1.5 & 1.7 \\
\hline \multirow{4}{*}{159.9} & -0.5 & -0.6 & 0.5 & 0.5 & -0.5 & -0.4 & 0.5 & 0.4 & -0.5 & -0.4 & 0.5 & 0.7 \\
\hline & -1.0 & -1.2 & 1.0 & 1.0 & -1.0 & -1.0 & 1.0 & 0.8 & -1.0 & -1.0 & 1.0 & 1.0 \\
\hline & -1.5 & -1.7 & 1.5 & 1.3 & -1.5 & -1.4 & 1.5 & 1.2 & -1.5 & -1.5 & 1.5 & 1.6 \\
\hline & -0.5 & -0.6 & 0.5 & 0.5 & -0.5 & -0.5 & 0.5 & 0.4 & -0.5 & -0.6 & 0.5 & 0.5 \\
\hline \multirow{2}{*}{179.9} & -1.0 & -1.2 & 1.0 & 0.9 & -1.0 & -1.0 & 1.0 & 0.8 & -1.0 & -1.1 & 1.0 & 1.0 \\
\hline & -1.5 & -1.7 & 1.5 & 1.3 & -1.5 & -1.5 & 1.5 & 1.3 & -1.5 & -1.6 & 1.5 & 1.5 \\
\hline
\end{tabular}

Table 2 The expected (exp.) and observed (obs.) lateral shifts obtained in the anterior portal image.

\begin{tabular}{|c|c|c|c|c|}
\hline \multirow{2}{*}{$\begin{array}{l}\text { SID } \\
\text { (cm) }\end{array}$} & \multicolumn{2}{|c|}{ Left lateral shift $(\mathrm{cm})$} & \multicolumn{2}{|c|}{ Right lateral shift $(\mathrm{cm})$} \\
\hline & expected & observed & expected & Observed \\
\hline \multirow{3}{*}{140.0} & -0.5 & -0.6 & 0.5 & 0.3 \\
\hline & -1.0 & -1.2 & 1.0 & 1.1 \\
\hline & -1.5 & -1.8 & 1.5 & 1.6 \\
\hline \multirow{3}{*}{149.9} & -0.5 & -0.5 & 0.5 & 0.5 \\
\hline & -1.0 & -1.1 & 1.0 & 1.0 \\
\hline & -1.5 & -1.7 & 1.5 & 1.6 \\
\hline \multirow{4}{*}{159.9} & -0.5 & -0.6 & 0.5 & 0.5 \\
\hline & -1.0 & -1.0 & 1.0 & 1.0 \\
\hline & -1.5 & -1.8 & 1.5 & 1.4 \\
\hline & -0.5 & -0.6 & 0.5 & 0.5 \\
\hline \multirow{2}{*}{179.9} & -1.0 & -1.1 & 1.0 & 1.0 \\
\hline & -1.5 & -1.7 & 1.5 & 1.6 \\
\hline
\end{tabular}




\section{Evaluation of positional accuracy of epid using imrt graticule phantom in extended source to imager distance setups: formalism of qa}

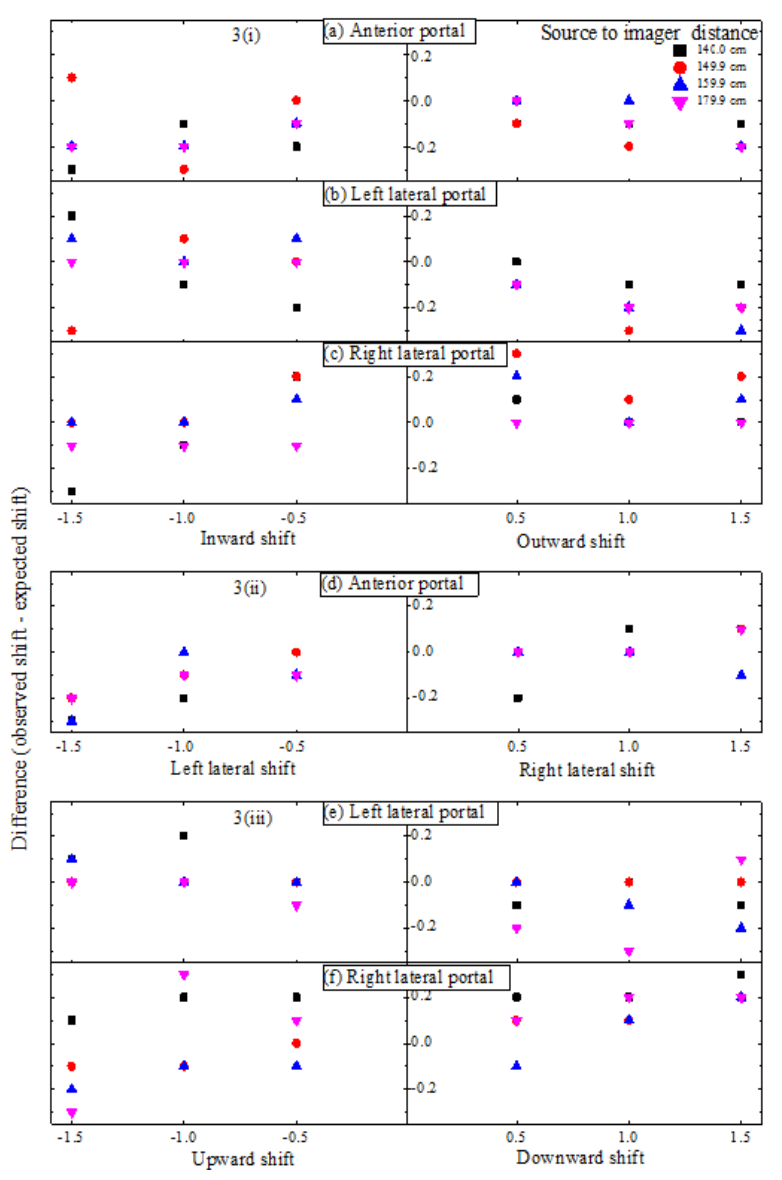

Fig. 3 The graphical representation shows the expected shift in longitudinal, lateral and vertical directions verses difference between the expected and observed shift at varying SID (all units are in $\mathrm{cm}$ ). orientations. Rowshanfarzad et al. ${ }^{[25]}$ found that the sag in EPID was found to be $0.3 \mathrm{~mm}$ and $2.5 \mathrm{~mm}$ in cross-plane and in-plane direction. Although the large deviation was observed for in-plane as that of cross plane direction and the accepted criterion for non-stero tactic linac is $2 \mathrm{~mm}$ as per AAPM TG 142 report ${ }^{[26]}$. The on-board imaging of patients suffering from different types of tumors is done at different imager distances. This is because of limitation of imaging system, location of tumor and coverage of larger target volumes. For example, the patient suffering from cervix carcinoma and having anterior posterior separation more than $30 \mathrm{~cm}$, the image cannot be acquired at $140 \mathrm{~cm}$. This is due to more possibility of collision between imager and the treatment couch. Similarly for the same patient, it is difficult to acquire image in the lateral direction for same SID. The same problem was also observed for peripheral (non-centric) tumors. In cranio-spinal irradiation, brain was treated with lateral opposed isocentric fields with collimator rotation and isocentric table rotation and a single dorsal field is not sufficient to cover the whole spine for adult patients. In order to cover the whole spine, source to surface distance was increased as compared to the routine treating distances [27]. Therefore, the imaging was done by EPID for such patients at extended imager positions. These setups may require verification of, e.g., reproducibility of change in source to imager distance, sag in the gantry during rotation therapy, sag in the carriage mounted in the head of the Linac unit to accommodate the different accessory, e.g., MLC, blocks etc. In such setups, EPID plays very important role in terms of ease and less time consumption. The relation between expected and observed shift was well correlated and geometrical accuracy was maintained at all imager position.

Table 3 The expected and observed vertical shifts obtained in the lateral portal images at different SID.

\begin{tabular}{|c|c|c|c|c|c|c|c|c|}
\hline \multirow{3}{*}{$\begin{array}{c}\text { SID } \\
(\mathrm{cm})\end{array}$} & \multicolumn{4}{|c|}{ Left lateral portal image } & \multicolumn{4}{|c|}{ Right lateral portal image } \\
\hline & \multicolumn{2}{|c|}{ upward shift (cm) } & \multicolumn{2}{|c|}{ downward shift (cm) } & \multicolumn{2}{|c|}{ upward shift (cm) } & \multicolumn{2}{|c|}{ downward shift $(\mathrm{cm})$} \\
\hline & Expected & Observed & Expected & Observed & Expected & Observed & Expected & Observed \\
\hline \multirow{4}{*}{140.0} & -0.5 & -0.5 & 0.5 & 0.4 & -0.5 & -0.3 & 0.5 & 0.7 \\
\hline & -1.0 & -0.8 & 1.0 & 1.0 & -1.0 & -0.8 & 1.0 & 1.2 \\
\hline & -1.5 & -1.4 & 1.5 & 1.4 & -1.5 & -1.4 & 1.5 & 1.8 \\
\hline & -0.5 & -0.5 & 0.5 & 0.5 & -0.5 & -0.5 & 0.5 & 0.6 \\
\hline \multirow[t]{2}{*}{149.9} & -1.0 & -1.0 & 1.0 & 1.0 & -1.0 & -1.1 & 1.0 & 1.1 \\
\hline & -1.5 & -1.5 & 1.5 & 1.5 & -1.5 & -1.6 & 1.5 & 1.7 \\
\hline \multirow{3}{*}{159.9} & -0.5 & -0.5 & 0.5 & 0.5 & -0.5 & -0.6 & 0.5 & 0.4 \\
\hline & -1.0 & -1.0 & 1.0 & 0.9 & -1.0 & -1.1 & 1.0 & 1.1 \\
\hline & -1.5 & -1.4 & 1.5 & 1.3 & -1.5 & -1.7 & 1.5 & 1.7 \\
\hline \multirow{3}{*}{179.9} & -0.5 & -0.6 & 0.5 & 0.3 & -0.5 & -0.4 & 0.5 & 0.6 \\
\hline & -1.0 & -1.0 & 1.0 & 0.7 & -1.0 & -0.7 & 1.0 & 1.2 \\
\hline & -1.5 & -1.5 & 1.5 & 1.6 & -1.5 & -1.8 & 1.5 & 1.7 \\
\hline
\end{tabular}

\section{DISCUSSION}

In the present study, IMRT graticule phantom was used to develop QA program for retractable arm EPID. The phantom have no breathing motion, therefore the error between the image acquired by the EPID and reference (DRR) image is either due to inbuilt error within in the imaging system or personnel error while matching these two images or effect of EPID sag at different orientations w.r.t. phantom position. For sufficiently large difference between planned position and the treatment position, appropriate corrections have to be applied. The study was carried out to find the effect of sag in EPID due to gravity at extended imager distances and varying beam
The maximum error was obtained to be $3 \mathrm{~mm}$ and it occurs for almost all imager positions w.r.t. the beam direction. If the gravity sag is significant then it will show large variations for that particular imager orientation w.r.t radiation beam, i.e., right lateral and left lateral positions and extended imager position. The fine correlation between expected and observed error shows non-considerable effect of EPID sag due to gravity at the image acquisition.

\section{CONCLUSION}

The IMRT graticule phantom is a useful tool to check the geometrical accuracy and hence the QA of EPID. The method 
used in this study is very effective to check the mechanical characteristic and consistency of the retractable arm EPID (Varian Oncology Ltd) and can be performed routinely for other supporting arm EPID. The effect of EPID sag due to gravity was not significant for detection of shift in the patient's position. The positional reproducibility of phantom with EPID (R-arm) was well maintained. Any slight variation can due to fluctuation in the position of EPID or personnel errors while matching the DRR and portal image obtained for a known shift at different SIDs. The close agreement between expected and measured shift at different distances and different orientations (gantry angles $0^{\circ}, 90^{\circ}$ and $270^{\circ}$ ) imply that EPID have proved effective tool for routine verification of patient setup during treatment in extended SID setups.

\section{References}

1. Marco Zaider and Leonid Hanin. Tumor control probability in radiation treatment. Med Phys. 2011; 38: 574.

2. Gregoire V. Tumor control probability (TCP) and normal tissue complication probability (NTCP) in head and neck cancer. Rays. 2005; 30(2):105-108.

3. Baumann M and Petersen C. TCP and NTCP: A basic introduction. Rays. 2005; 30(2): 99-104.

4. P. Kallman, A. Agren and A. Brahme. Tumour and normal tissue responses to fractionated non-uniform dose delivery. Int J Radiat Biol. 1992; 62(2): 249-262.

5. S. A. Rosenthal, J. M. Galvin, J. W. Goldwein, A. R. Smith and P. H. Blitzer. Improved methods for determination of variability in patient positioning for radiation therapy using simulation and serial portal film measurements. Int J Radiat Oncol Biol Phys. 1992; 23 (3): 621-625.

6. Van Elmpt W, McDermott L, Nijsten S, Wendling M, Lambin P and Mijnheer B. A literature review of electronic portal imaging for radiotherapy dosimetry. Radiother and Oncol. 2008; 88:289-309.

7. Ann Van Esch, Tom Depuydt and Dominique Pierre Huyskens. The use of an aSi-based EPID for routine absolute dosimetric pre-treatment verification of dynamic IMRT fields. Radiother and Oncol. 2004; 71:223-234.

8. S C Vieira, M L P Dirkx, K L Pasma and B J M Heijmen. Dosimetric verification of X-ray fields with steep dose gradients using an electronic portal imaging devic. Phys Med. Biol. 2003; 48:157-166.

9. Marion Essers and BenJMijnheer. In vivo dosimetry during external photon beam radiotherapy. Int J Radiat Oncol Biol Phys.1999;43:245-259.

10. M.G.Herman, J.J. Kruse and C.R. Hagness. Guide to clinical use of electronic portal imaging. J Appl Clin Med Phy. 2000; 1(2):38-57.

11. I.J.Das, M. Cao, C.W. Cheng, V.Misic, K.Scheuring, E.Scdule and P.A. Johnstone. A QA phantom for electronic portal imaging devices. J Appl Clin Med Phy. 2011; 12(2): 3350.
12. J.E. Marks, A.G. Haus, H.G. Sutton, and M.L. Griem. The value of frequent treatment verification films in reducing localization error in the irradiation of complex fields. Cancer. 1976; 37: 2755-2761.

13. Birendra Kumar Rout, Mukka Chandra Shekar, Alok Kumar and Kondapalli Kesava Durga Ramesh. Quality control test for electronic portal imaging device using QC-3 phantom with PIPSpro. Int J Cancer Ther Oncol. 2014; 2(4):1-6.

14. BaozhouSun, S.MurtyGoddu, Sridhar Yad dan ap udi, Camille Noel,Hua Li, Bin Cai, James Kavanaugh and Sasa Mutic. Daily QA of linear accelerators using only EPID and OBI. Med. Phys. 2015; 42: 5584.

15. A. L. Boyer, L. Antonuk, A. Fenster, M. Van Herk, H. Meertens, P. Munro, L. E. Reinstein and J. Wong. A review of electronic portal imaging devices (EPIDs). Med Phys. 1992; 19 (1):1-16.

16. S. Webb. The Physics of Three Dimensional Radiation Therapy. 1st ed. Bristol, UK, IOP Ltd.; 1993.

17. H. Roehrig and C. Cheng. Real-time imaging detectors for portal imaging. Proc.SPIE2009 1993, 144-167.

18. P. Munro. Portal Imaging Technology: Past, Present and Future. Seminars in Radiation Oncology 1995; 5: 115-133.

19. F. M. Khan. The Physics of Radiation Therapy. $4^{\text {th }}$ ed. Philadelphia, USA: Lippi. William and Wilkins; 2010.

20. D. Greene. And P. C. Williams. Linear Accelerators for radiation therapy. $2^{\text {nd }}$ ed. Madison Avenue, NY: Taylor \& Francis Group, 1997.

21. Anders Gustafsson, Bengt K. Lind and Anders Brahme. A generalized pencil beam algorithm for optimization of radiation therapy. Med Phy. 1994; 21: 343.

22. Martin Soukup, Matthias Fippeland Markus Alber. A pencil beam algorithm for intensity modulated proton therapy derived from Monte Carlo simulations. Phys Med Biol. 2005; 50: 5089-5104.

23. Christopher M. Bragg and John Conway. Dosimetric verification of the anisotropic analytical algorithm for radiotherapy treatment planning. Radiother Oncol. 2006; 81:315-323.

24. Christopher M. Bragg, Katrina Wingate and John Conway. Clinical implications of the anisotropic analytical algorithm for IMRT treatment planning and verification. Radiother Oncol. 2008; 86: 276-284.

25. Piotr Milecki, Sergiusz Nawrocki, Julian Malicki, Grazyna Stryczynska. Evaluation of an electronic portal imaging device (target view, ge) as a quality assurance tool. Reports of Practical Oncology \& Radiotherapy 2001; 6:169-172.

26. Coen W. Hurkmans, Peter Remeijer, Joos V. Lebesque and Ben J. Mijnheer. Set-up verification using portal imaging; review of current clinical practice. Radiother Oncology. 2001; 58: 105-120.

27. Klein E E, Hanley J, Bayouth J. Task group 142 report: Quality assurance of medical accelerator. Med. Phys. 2009; 36: 4197-4112.

\section{Please cite this article in press as:}

Ranjit Singh., Teerthraj Verma., Harpreet Singh Kainth., Chappe Ram., Devinder Mehta., Bhupinder S. Rana., Jhangvir Singh Shahi and Baljinder Singh (2017), Evaluation of Positional Accuracy of Epid Using Imrt Graticule Phantom In Extended Source to Imager Distance Setups: Formalism of Qa', International Journal of Current Advanced Research, 6(3), pp. 2389-2393.

http://dx.doi.org/10.24327/ijcar.2017. 2393.0015 